\title{
The Suppressive Field of Neurons in Lateral Geniculate Nucleus
}

\author{
Vincent Bonin, ${ }^{1,2}$ Valerio Mante, ${ }^{1,2}$ and Matteo Carandini ${ }^{1,2}$ \\ ${ }^{1}$ Smith-Kettlewell Eye Research Institute, San Francisco, California 94115, and ${ }^{2}$ Institute of Neuroinformatics, University of Zurich and Swiss Federal \\ Institute of Technology, $\mathrm{CH}-8057$ Zurich, Switzerland
}

The responses of neurons in lateral geniculate nucleus (LGN) exhibit powerful suppressive phenomena such as contrast saturation, size tuning, and masking. These phenomena cannot be explained by the classical center-surround receptive field and have been ascribed to a variety of mechanisms, including feedback from cortex. We asked whether these phenomena might all be explained by a single mechanism, contrast gain control, which is inherited from retina and possibly strengthened in thalamus. We formalized an intuitive model of retinal contrast gain control that explicitly predicts gain as a function of local contrast. In the model, the output of the receptive field is divided by the output of a suppressive field, which computes the local root-mean-square contrast. The model provides good fits to LGN responses to a variety of stimuli; with a single set of parameters, it captures saturation, size tuning, and masking. It also correctly predicts that responses to small stimuli grow proportionally with contrast: were it not for the suppressive field, LGN responses would be linear. We characterized the suppressive field and found that it is similar in size to the surround of the classical receptive field (which is eight times larger than commonly estimated), it is not selective for stimulus orientation, and it responds to a wide range of frequencies, including very low spatial frequencies and high temporal frequencies. The latter property is hardly consistent with feedback from cortex. These measurements thoroughly describe the visual properties of contrast gain control in LGN and provide a parsimonious explanation for disparate suppressive phenomena.

Key words: cat; gain control; receptive field; masking; adaptation; inhibition

\section{Introduction}

The visual responses of neurons in lateral geniculate nucleus (LGN) reveal suppressive phenomena that cannot be explained by the classical center-surround receptive field. First, responses saturate when stimulus contrast increases [contrast saturation (Derrington and Lennie, 1984; Chino and Kaplan, 1988; Sclar et al., 1990; Kremers et al., 2001; Alitto and Usrey, 2004)]. Second, responses to a stimulus are reduced by superimposition of another [masking (Cudeiro and Sillito, 1996; Felisberti and Derrington, 1999; Freeman et al., 2002; Solomon et al., 2002)]. Third, responses decrease when stimulus size is increased beyond an optimal value [size tuning (Cleland et al., 1983a; Murphy and Sillito, 1987; Jones and Sillito, 1991; Solomon et al., 2002; Ozeki et al., 2004)]. These three phenomena are suppressive, because responses are smaller than would be expected from the classical center-surround receptive field alone.

There is little agreement as to the physiological basis of these suppressive phenomena. Some have ascribed suppression to inhibitory signals within thalamus (Hubel and Wiesel, 1961; Levick

Received May 6, 2005; revised Sept. 30, 2005; accepted 0ct. 9, 2005.

This work was performed in 2001-2002 in Zurich, supported by the Swiss National Science Foundation, and in 2003-2004 in San Francisco, supported by the James S. McDonnell Foundation 21st Century Research Award in Bridging Brain, Mind, and Behavior. We thank Séverine Durand for help in the experiments and Samuel Solomon and Robert Frazor for comments on this manuscript.

Correspondence should be addressed to Matteo Carandini, Smith-Kettlewell Eye Research Institute, 2318 Fillmore Street, San Francisco, CA 94115. E-mail address: matteo@ski.org.

DOI:10.1523/JNEUROSCI.3562-05.2005

Copyright $\odot 2005$ Society for Neuroscience 0270-6474/05/2510844-13\$15.00/0 et al., 1972; Cleland et al., 1983a; Sclar, 1987; Felisberti and Derrington, 1999). Others attributed it to cortical feedback (Murphy and Sillito, 1987; Przybyszewski et al., 2000; Webb et al., 2002; Alitto and Usrey, 2003).

Moreover, there is little agreement as to how the suppressive phenomena should be explained functionally: how should the receptive field model be extended to account for response suppression? Contrast saturation has been described by a compressive relationship between stimulus intensity and cell output (Derrington and Lennie, 1984; Sclar et al., 1990; Felisberti and Derrington, 1999; Przybyszewski et al., 2000; Kremers et al., 2001; Solomon et al., 2002; Webb et al., 2002; Alitto and Usrey, 2004). Masking has been described by a divisive gain control mechanism (Freeman et al., 2002; Solomon et al., 2002). Size tuning has been attributed to a large subtractive region surrounding the receptive field (Solomon et al., 2002; Nolt et al., 2004; Ozeki et al., 2004). These proposals aim to account for one suppressive phenomenon at a time and not for all suppressive phenomena at once. Moreover, they fail to explain known relationships between suppressive phenomena. For example, size tuning depends on contrast, being strong at high contrast and absent at low contrast (Solomon et al., 2002; Ozeki et al., 2004).

We suggest that the suppressive phenomena are parsimoniously explained by a single mechanism of contrast gain control, which operates in retina and is possibly strengthened in thalamus. Indeed, the stimulus manipulations that elicit the suppressive phenomena invariably increase local contrast. Increasing lo- 


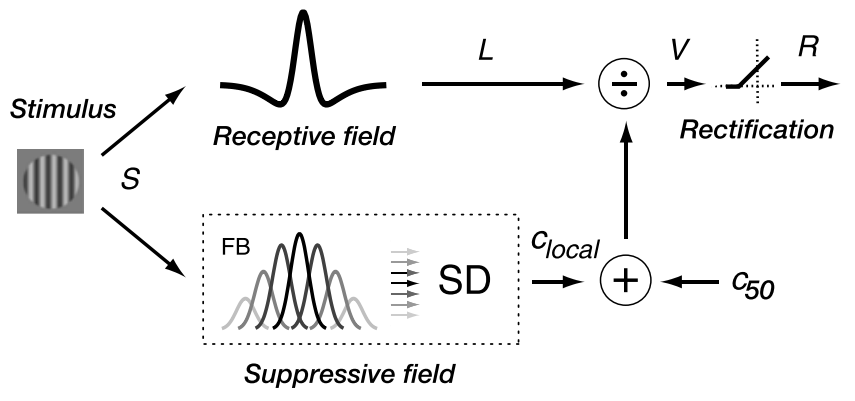

Figure 1. Model of LGN responses. The model includes a receptive field and a suppressive field. The receptive field has the classical center-surround organization (difference-ofGaussians). The suppressive field computes the SD of the outputs of a Gaussian-weighted bank of filters (FB) and sums the result to a constant, $c_{50}$. The signals from receptive field and suppressive field meet at a divisive stage. The output of the division is then rectified to yield positive firing rates.

cal contrast decreases the responsiveness (gain) of retinal ganglion cells (Shapley and Victor, 1978, 1981; Victor, 1987; Baccus and Meister, 2002). This effect is thought to be enhanced in LGN (Kaplan et al., 1987; Sclar, 1987; Cheng et al., 1995). It could potentially explain the suppressive phenomena.

\section{Materials and Methods}

We characterized the responses of 34 well isolated neurons recorded in LGN of eight anesthetized, paralyzed cats. These neurons were held long enough ( $>2 \mathrm{~h}$, commonly $4 \mathrm{~h})$ to perform a series of more than six experiments, which involved $>180$ stimuli.

Recording. Adult cats were anesthetized with ketamine $(20 \mathrm{mg} / \mathrm{kg})$ mixed with acepromazine $(0.1 \mathrm{mg} / \mathrm{kg})$ or xylazine $(1 \mathrm{mg} / \mathrm{kg})$. Anesthesia was maintained with a continuous intravenous infusion of penthotal $\left(0.5-4 \mathrm{mg} \cdot \mathrm{kg}^{-1} \cdot \mathrm{h}^{-1}\right)$. Animals were paralyzed with pancuronium bromide $\left(0.15 \mathrm{mg} \cdot \mathrm{kg}^{-1} \cdot \mathrm{h}^{-1}\right)$ and artificially respired with a mixture of $\mathrm{O}_{2}$ and $\mathrm{N}_{2} \mathrm{O}$ (typically 1:2). EEG, electrocardiogram, and end-tidal $\mathrm{CO}_{2}$ were continuously monitored. Extracellular signals were recorded with quartz-coated platinum/tungsten microelectrodes (Thomas Recording, Giessen, Germany), sampled at $12 \mathrm{kHz}$, and stored for spike discrimination. A craniotomy was performed to access the right LGN (at approximately Horsley-Clarke A6L9), whose location was determined from the sequence of ocular preference changes along the penetration. Cells had receptive fields with eccentricities ranging from $2^{\circ}$ to $45^{\circ}$, with an average value of $13.4 \pm 8.9^{\circ}(\mathrm{SD} ; n=34)$. All procedures were approved by the Veterinary Office of Canton Zurich and by the Animal Care and Use Committee of the Smith-Kettlewell Eye Research Institute.

Visual stimuli were displayed using the Psychophysics Toolbox (Brainard, 1997; Pelli, 1997) and presented monocularly on a calibrated monitor with mean luminance of $32 \mathrm{~cd} / \mathrm{m}^{2}$ and refresh rate of $124 \mathrm{~Hz}$. Stimuli lasted 1-4 s and were repeated three to six times (blocks). Stimulus order within blocks was randomized. Each block included one or more blank stimuli.

We classified cells into $\mathrm{X}$ and $\mathrm{Y}$ types from responses to counterphasemodulated sinusoidal gratings of different spatial frequencies (EnrothCugell and Robson, 1966; Hochstein and Shapley, 1976). Grating phase was chosen to elicit the smallest first harmonic response. Cells are classified as $\mathrm{Y}$ type if the amplitude of the second harmonic reliably exceeded that of the first harmonic. Most units (28 of 34) were of the X type.

Stimuli were centered on the receptive field based on responses to drifting gratings enclosed in small circular apertures displayed at different spatial locations. Control measurements were repeated regularly to ensure correct stimulus centering in the face of the drifts in eye position that can occur even during paralysis. When such drifts occurred, we added a new parameter to the model: stimulus offset from the center. We obtained its value by fitting responses to stimuli of different diameters. We then held this value fixed across experiments starting with the experiment in which the shift occurred.
Model. As illustrated in Figure 1, the model contains two pathways, one linear and one nonlinear.

The linear pathway involves a classical center (ctr) - surround (srd) receptive field, a difference-of-Gaussians, $\mathrm{RF}=G_{\mathrm{ctr}}-k_{\mathrm{srd}} G_{\text {srd }}$, where $G_{\text {ctr }}$ and $G_{\text {srd }}$ are two-dimensional Gaussian densities of width $\sigma_{\text {ctr }}$ and $\sigma_{\text {srd }}$, and $k_{\text {srd }}$ is the relative weight of the surround. The receptive field is convolved with the stimulus $S(x, y, t)$ to produce a linear response:

$$
L(t)=[S * \operatorname{RF}](0,0, t) .
$$

For our stimuli, which consist of the sum of a "test" and a "mask" drifting gratings, $L(t)$ is the sum of the responses to the individual gratings:

$$
L(t)=L_{\text {test }}(t)+\alpha_{\text {mask }} L_{\text {mask }}(t)
$$

where $\alpha_{\text {mask }}$ denotes the relative effectiveness of the temporal frequency of the mask in driving the receptive field.

The nonlinear pathway involves a suppressive field that computes the $\mathrm{SD}$ of local luminance $c_{\text {local }}$. Assuming that the stimulus $S(x, y, t)$ has a mean of zero, local contrast follows

$$
c_{\text {local }}=\sqrt{\iiint S^{\prime}(x, y, t)^{2} G_{\mathrm{SF}}(x, y) d x d y d t},
$$

where $G_{\mathrm{SF}}$ denotes the suppressive field, a Gaussian of width $\sigma_{\mathrm{SF}}$, and $S^{\prime}(x, y, t)$ denotes the stimulus processed through a bank of filters, [ $S^{*}$ $H](x, y, t)$. We model each of these filters as a difference-of-Gaussians: $H=G_{u}-k_{d} G_{d}$.

The suppressive field controls neural gain by dividing the output of the receptive field. The result is

$$
V(t)=V_{\max } \frac{L(t)}{c_{50}+c_{\text {local }}},
$$

where $c_{50}$ determines the strength of the suppressive field, and $V_{\max }$ captures the overall responsiveness of the neuron.

Firing rate is a rectified version of $V$, with threshold $V_{0}$ :

$$
R(t)=\left[V(t)-V_{0}\right]_{+} .
$$

Model characterization. We fitted the model onto the harmonic components of the spike trains at the temporal frequencies of the stimuli. Let $\left\{t_{i}\right\}$ denote the spike times of a response to a stimulus of temporal frequency $f ;$ the harmonic component follows $r=1 / N\left\|\sum_{j} \exp \left[-2 \pi i f t_{j}\right]\right\|$, where $\|x\|$ denotes the modulus of the complex number $x$, and $N$ is the number of spikes.

Fits minimize the square error between responses of neuron and model $\Sigma_{i j}\left(r_{i j}-m_{j}\right)^{2}$, where $r_{i j}$ denotes the response of a neuron to trial $i$ of stimulus $j$, and $m_{i}$ is the response predicted by the model.

To estimate model parameters, we fitted Equations $1-5$ to a sequence of four experiments, each constraining one or more parameters. The first experiment constrained the parameters of the receptive field ( $\sigma_{\mathrm{ctr}}, \sigma_{\mathrm{srd}}$, and $k_{\text {srd }}$ ) and involved drifting gratings varying in spatial frequency. Gratings had $50 \%$ contrast, optimal temporal frequency, $>20^{\circ}$ in diameter, and one of $>14$ logarithmically spaced spatial frequencies. The subsequent three experiments constrained the parameters of the suppressive field $\left(c_{50}, \sigma_{\mathrm{SF}}, \sigma_{u}, \sigma_{d}, k_{d}\right.$, and $\left.\alpha_{\text {mask }}\right)$ and involved sums of a test grating and a mask grating, in which we varied mask contrast (see Fig. $3 A, D$ ), mask diameter (see Fig. $3 B, E$ ), and mask spatial frequency (see Fig. 3C,F). Test diameter, spatial frequency and temporal frequency were optimized cell by cell to elicit maximal response. Unless varied, the attributes of the mask were also optimal for the cell. Each experiment included test gratings presented alone at more than six contrasts and sums of a $50 \%$ contrast test and a mask of variable attributes. Parameter estimates obtained when fitting one experiment were held fixed in fits to subsequent experiments. We repeated this sequence of fits until parameters changed by $<1 \%$ since the previous sequence iteration. The result was a single set of parameters used to predict responses to all experiments.

Parameters $V_{\max }$ and $V_{0}$ were allowed to vary across experiments to account for slow changes in neural responsiveness and spontaneous ac- 
A

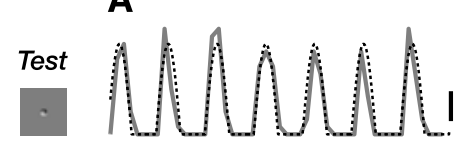

C
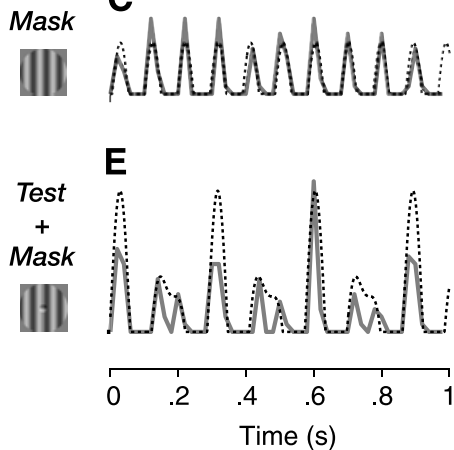

B

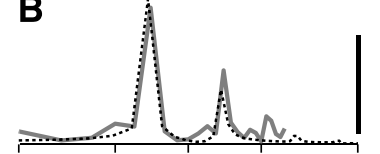

D

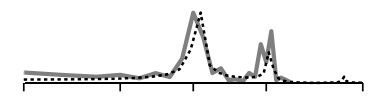

$\mathbf{F}$

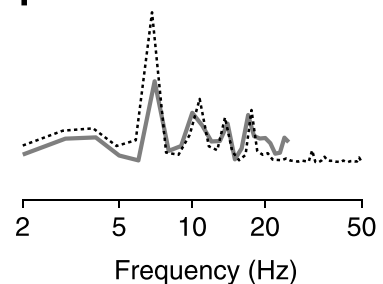

Figure 2. Masking experiments. Solid curves are firing rate responses of an example neuron. Dotted curves are predictions of receptive field followed by rectification. $A$, Responses to test grating presented alone. $\boldsymbol{B}$, Same, but showing amplitude spectrum of responses. $\boldsymbol{C}, \boldsymbol{D}$, Measured and predicted responses to mask alone. $\boldsymbol{E}, \boldsymbol{F}$, Measured and predicted responses to sum of test and mask. Calibration bars in $\boldsymbol{A}$ and $\boldsymbol{B}$ indicate 50 spikes/s. Cell 44.4.2.

tivity. During the course of a recording session with a given neuron (which lasted on average $289 \pm 72 \mathrm{~min}, \mathrm{SD} ; n=34$ ), the spontaneous firing rate varied over $18 \pm 11$ spikes/s, with variations $>25$ spikes $/ \mathrm{s}$ in 9 of 34 neurons. Similarly, the overall responsiveness varied substantially over time. The harmonic response to a $50 \%$ contrast stimulus varied on average by $25 \pm 16$ spikes/s, with variations of $>40$ spikes/s in 4 of 34 neurons. These variations in spontaneous firing rate and responsiveness were not statistically correlated $(p>0.2)$. We tracked them by letting $V_{\max }$ and $V_{0}$ vary from one experiment to another. In each experiment, we fixed $V_{0}$ to minus the mean spontaneous firing rate, which we obtained from the responses to blank stimuli, and we chose $V_{\max }$ to minimize square error between model and data. Had we fixed $V_{\max }$ and $V_{0}$ across experiments, the quality of predictions would have been compromised in many neurons.

Masking experiments. The logic of the masking experiments is that the test elicits strong responses, and the mask probes how the suppressive field influences these responses (Fig. 2). In general, both test (Fig. 2A, $B$ ) and mask (Fig. 2C,D) elicit responses when presented alone. These responses oscillate at the drift frequency and are well predicted by a simple model consisting of a receptive field followed by rectification (Fig. $2 A-D$, dotted curves). We chose the drift rates of test and mask to be incommensurate (e.g., 7.8 and $12.5 \mathrm{~Hz}$ ) so that, even when test and mask are superimposed (Fig. 2E,F), we can distinguish a "test response" (the component of the response at the test frequency) and a "mask response" (the component at the mask frequency). The average temporal frequencies of test and mask were $7.3 \pm 2.5$ and $12.6 \pm 2.9 \mathrm{~Hz}(n=34)$.

In the presence of the mask, the test response is smaller than predicted by the receptive field and rectification (Fig. $2 E, F$, dotted curves). This masking effect is explained by the full model because adding the mask increases the output of the suppressive field. From the response suppression caused by the mask, we deduce the degree to which the mask drives the suppressive field.

We obtained one parameter of the receptive field, $\alpha_{\text {mask}}$, from the mask responses and all parameters of the suppressive field from the test responses. We estimated the strength of the suppressive field $c_{50}$ by varying mask contrast over more than six logarithmically spaced values (Fig. $3 A, D)$. We estimated the size of the suppressive field $\sigma_{\mathrm{SF}}$ by varying mask diameter over $>10$ logarithmically spaced values (Fig. $3 B, E$ ). We estimated the parameters that describe the filter bank $\left(\sigma_{u}, \sigma_{d}\right.$, and $\left.k_{d}\right)$ by varying mask spatial frequency over $>10$ logarithmically spaced values (Fig. 3C,F). Finally, we used the mask responses to constrain $\alpha_{\text {mask }}$ (Eq.
2). We estimate $\alpha_{\text {mask }}$ from data in which mask contrast is varied (Fig. $3 D$ ) and use the same value for the remaining experiments (Fig. $3 E, F$ ).

Model evaluation. To judge the quality of model fits, we calculated the percentage of the variance in the averaged data that is explained by the model. This quantity is given by $100\left(1-n^{-1} \sum_{j}\left(\bar{r}_{j}-m_{j}\right)^{2} / \operatorname{var}\left[\bar{r}_{j}\right]\right)$, where $\bar{r}_{j}$ is the response to stimulus $j$ averaged across trials, and $n$ is the number of stimuli.

We tested model predictions with drifting gratings varying in contrast and diameter (see Fig. 5). This experiment included $>50$ stimuli covering a full matrix of more than six contrasts and more than eight diameters distributed on a logarithmic scale. The spatial frequency and temporal frequency of the gratings were set to the values eliciting maximal response. Parameters $V_{\max }$ and $V_{0}$ were left free to account for changes in neural responsiveness. The remaining parameters were held fixed to their previously estimated values.

To compare data and model predictions, we used two independent methods. First, to investigate the ability of the model to predict the effects of contrast on the size tuning curves, we fitted a difference-of-Gaussians to the tuning curves at each contrast (Sceniak et al., 1999). This function provided excellent fits (Ozeki et al., 2004). From these fits, we calculated the preferred stimulus diameter and the degree of size tuning (expressed as a fraction of the peak responses) and compared results for data and for model predictions. Second, to investigate the ability of the model to predict the effects of stimulus size on contrast saturation, we fitted a power law to the contrast-response curves at each diameter. The power law provided excellent fits, so the power-law exponent $\beta$ captures the linearity of the growth of responses with contrast: a value close to 0 indicates strong saturation, and a value close to 1 indicates linearity.

To measure the precision of model predictions for individual stimuli, we calculated $z$-scores of deviations between measured and predicted responses. The $z$-score of deviations is given by $z=(\bar{r}-m) / \hat{v}$, where $\hat{v}$ is a robust estimate of the SD of responses calculated across trials. To estimate $\hat{v}$, we fitted a power law relating the observed SD to the mean $\bar{r}$ and used the resulting predictions as estimate of SD.

Properties of the suppressive field. We estimated the sizes of receptive field and suppressive field from model parameters. We defined size as the diameter of the stimulus eliciting $95 \%$ of the maximum response. The receptive field center and surround sizes are given by $2 \sigma_{\text {ctr }} \sqrt{-2 \log (1-\eta)}$ and $2 \sigma_{\text {srd }} \sqrt{-2 \log (1-\eta)}$, where $\eta=0.95$. The suppressive field size is given by $2 \sigma_{\text {srd }} \sqrt{-2 \log \left(1-\eta^{2}\right)}$. These expressions differ because the Gaussians underlying the receptive field operate on stimulus intensity, whereas that underlying the suppressive field operates on stimulus energy.

To obtain independent measures of receptive field size, we recorded the responses to contrast-modulated circular annuli $(n=15$, in two additional animals) and varied annulus inner diameter (see Fig. 7C, symbols). The harmonic responses yield an annulus tuning curve that reflects the center-surround organization of the receptive field (Kilavik et al., 2003; Kremers et al., 2004). Responses are small for small inner diameter, because the annulus covers both the receptive field center and surround. Responses increase as the overlap between the annulus and the receptive field center decreases and reach a maximum when the annulus fills the receptive field surround. Responses then fall off as the annulus exits the receptive field surround. The inner diameter for which responses reach the floor (within \pm 1 SD of the response to a blank stimulus) indicates the size of the receptive field surround (see Fig. $7 C$, open arrow). We compared the estimates of receptive field size obtained with this method with those obtained from the spatial frequency tuning of responses and found them consistent.

We used model fits to compare the selectivity of receptive field and suppressive field for spatial frequency. We calculated the low- and highfrequency cutoffs of the outputs of receptive field and suppressive field. We defined cutoff as the frequency at which the outputs reach $50 \%$ of their maximum power ( $71 \%$ of the maximum amplitude).

We used masking experiments to measure the selectivity of the suppressive field for temporal frequency and orientation (see Fig. 8). Experiments were similar to those described above in "Model characterization" but with masks of either more than seven incommensurate temporal frequencies or 12 orientations. We estimated the selectivity of 
A
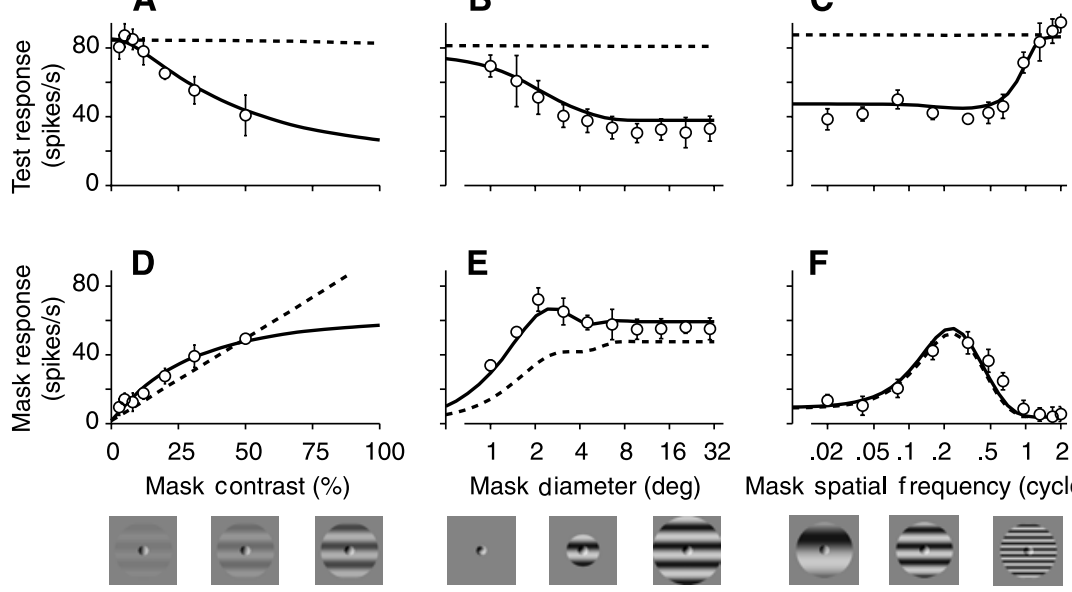

Mask diameter (deg) Mask
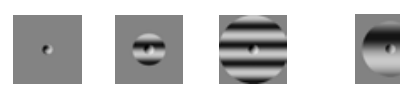

Figure 3. Masking. Stimuli are sums of a test grating and a mask grating drifting with incommensurate temporal frequencies Responses are measured at the frequency of the test, $7.8 \mathrm{~Hz}$ (test response; $\boldsymbol{A}-\boldsymbol{C}$ ) and at the frequency of the mask, $12.5 \mathrm{~Hz}$ (mask response; $\boldsymbol{D}, \boldsymbol{F}$ ). Unless otherwise stated, in this figure and in subsequent ones, the error bars indicate $\pm 1 \mathrm{SD}$. Curves show model fit. Dashed lines indicate predictions of receptive field followed by rectification. $\boldsymbol{A}, \boldsymbol{D}$, Responses as function of mask contrast $\left(V_{\max }=273 ; V_{0}=-6\right) . \boldsymbol{B}, \boldsymbol{E}$, Responses as function of mask diameter $\left(V_{\max }=242 ; V_{0}=-6\right) . \boldsymbol{C}, \boldsymbol{F}$, Responses as function of mask spatial frequency $\left(V_{\max }=275 ; V_{0}=-4\right)$. Test and mask had spatial frequency of 0.24 cycles/deg (unless varied) and diameter of $1.4^{\circ}$ and $14.1^{\circ}$ (unless varied). Cell 33.1.3.

the suppressive field by subtracting the test responses measured with mask from the test response measured without mask, normalizing and averaging across cells. We estimated the preferences of the receptive field by normalizing and averaging mask responses.

\section{Results}

Our results are organized as follows. First, we devise a simple model of contrast gain control based on the established model of ganglion cell responses proposed by Shapley and Victor (Shapley and Victor, 1981; Victor, 1987). We extend this model to the domain of arbitrary spatial images, by specifying how local contrast should be integrated across space. Then we test the model on a large array of responses of cat LGN neurons and show that it captures response suppression. Finally, we measure the visual properties of contrast gain control and discuss how these properties constrain the possible sources of the suppressive signals.

\section{Model}

Our hypothesis is that LGN responses, and in particular the suppressive phenomena, can be explained by a model of contrast gain control such as the one proposed by Shapley and Victor for retinal ganglion cells (Shapley and Victor, 1981; Victor, 1987). In this model, the temporal responses of ganglion cells result from a cascade of low-pass and high-pass filters, whose time constants depend on contrast.

We reformulated the Shapley-Victor model to directly express the effect of contrast on response gain. In Appendix, we simplify the expressions of the model by concentrating on responses to stimuli that vary slowly, because these responses are most affected by gain control. We considered only the effects on response amplitude, ignoring the effects on response timing. The result is a single divisive stage, in which the output of the receptive field appears in the numerator and a measure of local contrast appears in the denominator:

$$
R \approx \frac{L}{c_{50}+c_{\text {local }}},
$$

where $R$ is the response of the neuron, $L$ is the response of the receptive field (which grows linearly with contrast), $c_{50}$ is a constant, and $c_{\text {local }}$ is local contrast. This expression resembles models of divisive normalization used in visual cortex (Albrecht and Geisler, 1991; Heeger, 1992; Carandini et al., 1997).

Shapley and Victor (1978) did not specify how to compute local contrast $c_{\text {local }}$ but revealed important constraints. First, $c_{\text {local }}$ is invariant to stimulus position within the receptive field. Second, $c_{\text {local }}$ is an "even function" of stimulus intensity (Victor, 1987) (i.e., one that is the same for stimuli that are lighter or darker than the mean luminance). Third, $c_{\text {local }}$ grows with stimulus area (Shapley and Victor, 1981).

Guided by these constraints, we assumed that local contrast $c_{\text {local }}$ is the SD of the luminance falling under a Gaussian region concentric with the receptive field. We call this region "suppressive field," because its properties match the term coined by Levick et al. (1972) to describe a region that (1) is engaged equally by light or dark stimuli and (2) suppresses the responses of a cell. The SD is proportional to root-mean-square contrast. It fulfills the above constraints, and it can be generalized to arbitrary images.

The resulting model contains two image-processing pathways, the receptive field and the suppressive field (Fig. 1). The two pathways meet at a divisive stage, in which the receptive field provides the numerator and the suppressive field provides the denominator. The resulting output $V$ is then rectified to obtain a positive firing rate $R$.

The receptive field operates linearly: its output involves a simple weighted sum of the image intensities, with weights given by the receptive field profile. We model the receptive field with a difference-of-Gaussians (Rodieck, 1965; Enroth-Cugell and Robson, 1966), which has three free parameters: the width of the center Gaussian, the width of the surround Gaussian, and the strength of the surround relative to the center.

The operation of the suppressive field, instead, is nonlinear, because it involves square root and squaring operations. To compute local contrast, in particular, the suppressive field (1) processes the image through a bank of filters ["subunits" (Shapley and Victor, 1978)], (2) weights the filter outputs by a twodimensional Gaussian, a window that represents the spatial extent of the suppressive field, and (3) computes the SD of the resulting signals.

We next show that, with a single set of parameters, the model predicts masking, size tuning, and contrast saturation. We obtain the parameters of the model by fitting a series of masking experiments and then hold those parameters fixed and show that they account for size tuning and contrast saturation.

\section{Masking}

The model predicts masking, the suppression observed when two stimuli are overlaid (Fig. 3). Masking occurs when either one grating extends in the region surrounding the other (Sillito et al., 1993; Felisberti and Derrington, 1999; Solomon et al., 2002) or two gratings are superimposed (Freeman et al., 2002). In our measurements, the two gratings are a fixed optimal test at $50 \%$ 
contrast and a mask whose attributes are varied. Test and mask have incommensurate temporal frequencies, which elicit distinct response components (Bonds, 1989): a test response that oscillates at the temporal frequency of the test and a mask response that oscillates at the frequency of the mask. We first investigate how test responses are affected by mask attributes.

The model predicts that adding a mask should reduce test responses. The predicted test responses are given by Equation 6 , in which the mask affects only the output of the suppressive field (the term $c_{\text {local }}$ in the denominator). Adding the mask, therefore, increases $c_{\text {local }}$ and thus reduces test responses.

We test the predictions of the model by varying mask contrast (Fig. $3 A$ ), diameter (Fig. 3B), and spatial frequency (Fig. 3C) and find that the model yields good fits (solid curves). (1) Increasing mask contrast progressively suppresses the test response (Fig. 3A). The model predicts this effect because increasing mask contrast increases the local contrast $c_{\text {local }}$. With masks of low contrast, there is little suppression because $c_{\text {local }}$ is dominated by the constant $c_{50}$. (2) Increasing mask diameter progressively suppresses test responses until responses reach a plateau (Fig. 3B). The model captures this decrease because increasing mask diameter increases local contrast $c_{\text {local }}$. The plateau is reached once the mask extends beyond the suppressive field. (3) Suppression depends on mask spatial frequency (Fig. 3C), being strong at low spatial frequencies and absent at high frequencies. The model captures this behavior because of the filters at the front end of the suppressive field, which endow the suppressive field with frequency preferences.

The suppressive field plays a critical role in explaining these observations. The fits performed without it (Fig. 3, dashed curves) are clearly inadequate, indicating that neither the receptive field nor the rectification stage can account for masking. The fits performed including the suppressive field, instead, have high quality: overall, the model explains $94.4 \%$ of the variance (median, $n=34)$.

The model can also predict the mask responses, the components of responses at the temporal frequency of the mask (Fig. $3 D-F)$. In this case, varying mask attributes varies the outputs of both receptive field and suppressive field. Mask responses display strong saturation (Fig. 3D), they are tuned for stimulus size (Fig. $3 E$ ), and their tuning for spatial frequency is bandpass (Fig. $3 F$ ). The resulting model predictions yield good fits to the mask responses (Fig. 3, solid curves). Fits of similar quality were obtained in the remaining cells, with the model explaining $87.9 \%$ of the variance (median, $n=34$ ).

\section{Estimating model parameters}

We use these masking experiments to constrain key model parameters, which we henceforth keep fixed. From the effects of mask contrast, we estimate the constant $c_{50}$ (Fig. $3 A$ ). From the effects of mask diameter, we estimate the width of the suppressive field $\sigma_{\mathrm{SF}}$ (Fig. $3 B$ ). From the effects of mask spatial frequency, we estimate the parameters of the filters at the front end of the suppressive field (Fig. $3 C$ ). The result is one set of parameters for each cell (Table 1).

The fits yielded reliable estimates of the parameters (Fig. 4). For most parameters, the values yielding the best fits fell in a narrow range. This is the case, for example, for the width of the receptive field surround $\sigma_{\text {srd }}$ (Fig. $4 A$ ), for the strength of suppressive field $c_{50}$ (Fig. $4 \mathrm{~B}$ ), and for the width of the suppressive field $\sigma_{\mathrm{SF}}$ (Fig. $4 C$ ). The exception to this rule is the strength of the surround of the subunits that compose the suppressive field, $k_{d}$ (Fig. $4 D$ ). For this parameter, any value $<0.8$ would be approximately equally good at fitting the responses, which have a lowpass behavior (Fig. 3B). Had suppression been bandpass, $k_{d}$ would have been constrained to the range $0.8-1.0$.

\section{Size tuning and contrast saturation}

Having characterized the model, we ask whether it predicts size tuning and contrast saturation. We test the model on responses to single drifting gratings whose diameter and contrast are varied independently. These data provide a stringent test, because they were not used to constrain the model parameters.

The model predicts size tuning and how this tuning depends on contrast (Fig. 5A). Size tuning is pronounced at high contrast (Fig. 5A, lighter symbols) but weak at low contrast (Fig. 5A, 

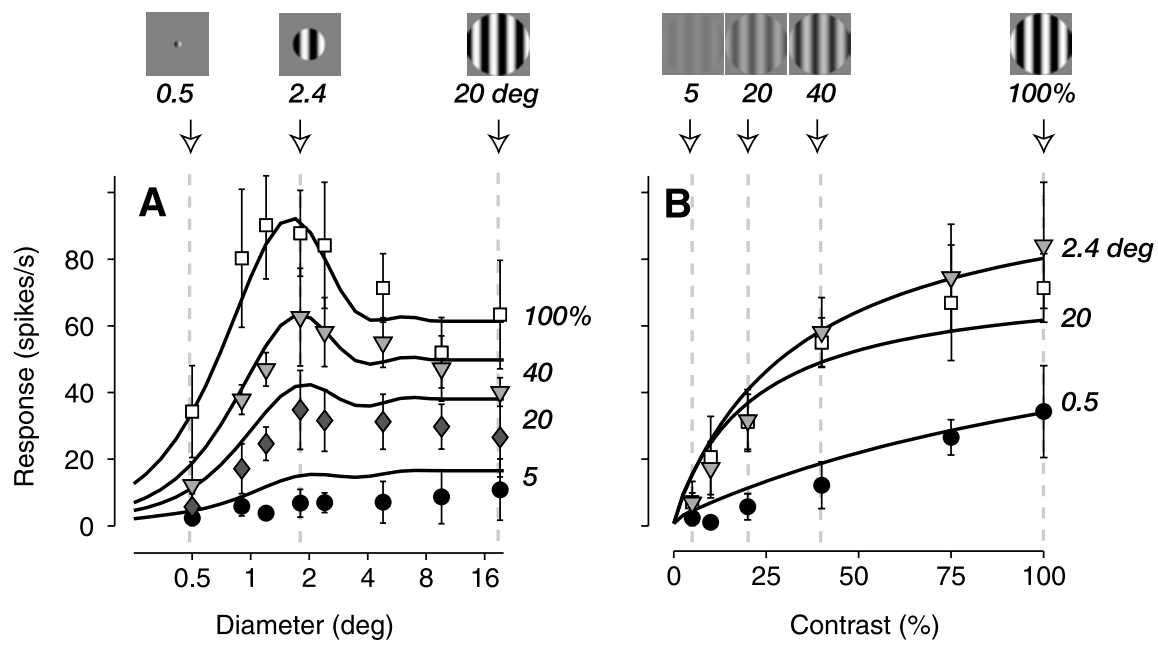

Figure 5. Size tuning and contrast saturation. Stimuli are gratings varying in diameter and contrast. Curves are predictions of model with parameters held fixed from previous measurements (Fig. $3 ; V_{\max }=128 ; V_{0}=-2$ ). $A$, Responses as a function of diameter, for selected contrasts. $\boldsymbol{B}$, Same data, plotted as a function of contrast, for selected diameters. Stimuli had optimal attributes: 0.24 cycles/deg and $7.8 \mathrm{~Hz}$. Cell 33.1.3 (93.9\% explained variance).

darker symbols) (Solomon et al., 2002; Ozeki et al., 2004). The model captures these effects (Fig. $5 A$, curves). It exhibits size tuning because extending the stimulus beyond the confine of the receptive field center increases the output of the suppressive field, $c_{\text {local }}$, but not the output of the receptive field. At low contrasts, instead, $c_{\text {local }}$ is much smaller than the constant $c_{50}$; the output of the suppressive field is ineffectual, and size tuning is not observed.

The dependence of size tuning on stimulus contrast is marked in our sample (Fig. 6A). In most cells, size tuning is strongest at high contrasts (Fig. 6A, open symbols) and weakest at low contrasts (Fig. 6A, filled symbols). To quantify the degree of size tuning, we estimate the amplitude of plateau responses, the responses to the largest stimulus tested, as a fraction of the peak responses. At $10 \%$ contrast, plateau responses are $27 \pm 20 \%$ smaller than the peak responses (mean $\pm \mathrm{SD} ; n=34$ ). The degree of size tuning increases with contrast and peaks at $100 \%$ contrast, at which plateau responses are $39 \pm 18 \%$ below peak responses. The model predicts a similar trend, with values of $11 \pm 9 \%$ at $10 \%$ contrast and $37 \pm 15 \%$ at $100 \%$ contrast.

A consequence of these effects is that preferred stimulus size decreases with contrast (Kremers et al., 2001; Solomon et al., 2002; Nolt et al., 2004). To quantify this effect, we estimate the diameter of the stimulus eliciting maximal response. This preferred stimulus diameter decreases as contrast is increased, ranging from $3.8 \pm 2.0^{\circ}$ at $10 \%$ contrast to $2.4 \pm 1.7^{\circ}$ at $100 \%$ contrast. The model captures this effect with predicted preferred diameters of $4.1 \pm 3.0^{\circ}$ at $10 \%$ contrast and of $2.5 \pm 1.6^{\circ}$ at $100 \%$ contrast.

The model also predicts how responses saturate with increasing contrast (Fig. $5 B$ ). Responses to large stimuli show strong saturation (Fig. $5 B$, lighter symbols). For these stimuli, the outputs of both receptive field and suppressive field are simply proportional to grating contrast $c$, so the model predicts that firing rate obeys $R \approx c /\left(c_{50}+c\right)$. This expression is known to capture the contrast responses of LGN neurons (usually contrast is elevated to a power of $n$, with $n$ close to 1 ) (Derrington and Lennie, 1984; Sclar et al., 1990; Felisberti and Derrington, 1999; Kremers et al., 2001).

The model predicts a novel phenomenon: that contrast satu- ration is pronounced only for large stimuli (Fig. 5B). Responses to small stimuli grow nearly linearly with contrast (Fig. $5 B$, darker symbols), whereas responses to large stimuli show clear saturation (Fig. $5 B$, lighter symbols). The model predicts linearity because small stimuli induce in the suppressive field a response that is negligible compared with the constant $\mathcal{c}_{50}$. These data suggest that there is nothing intrinsically nonlinear about LGN responses: were it not for the suppressive field, responses would grow linearly with contrast.

This pronounced dependence of saturation on stimulus size is seen throughout our sample (Fig. $6 B$ ). In most cells, saturation is strongest for large stimuli (Fig. $6 B$, white symbols), weaker for optimal stimuli (Fig. $6 B$, gray symbols), and nearly absent for the smallest stimuli (Fig. $6 B$, black symbols). To summarize the degree of saturation across cells, we fit a power law to the contrast-response curve at each stimulus diameter. Powerlaw exponents close to 0 indicate strong saturation; exponents close to 1 indicate linear growth. With an average exponent of $0.78 \pm 0.41$, saturation is weakest at the smallest stimulus diameter eliciting a reliable response. Saturation monotonically increases with stimulus diameter, with an average exponent of $0.46 \pm 0.18$ at the largest diameters tested. The model predicts the trend yielding exponents of $0.61 \pm 0.11$ for small diameters and $0.40 \pm 0.16$ for large diameters.

As expected from previous results (Shapley and Victor, 1978; Sclar, 1987), the effects of gain control appear stronger in Y cells than in X cells. The strength of the suppressive field $c_{50}$ averages $0.21 \pm 0.06$ in X cells $(n=28$, bootstrap estimates $)$ and $0.14 \pm$ 0.06 in Y cells $(n=6)$. The power-law fits of the contrast-response curve (at the largest stimulus tested) yielded exponents of $0.46 \pm 0.03$ in X cells and $0.40 \pm 0.10$ in Y cells. These differences, however, did not reach statistical significance (ANOVA, $p>0.1$ ).

There are also indications that gain control is more pronounced in ON-center cells than in OFF-center cells, as has been demonstrated in retina (Chander and Chichilnisky, 2001; Zaghloul et al., 2003). The constant $c_{50}$ averages $0.15 \pm 0.03(n=$ $19)$ in ON cells and $0.27 \pm 0.08$ in OFF cells $(n=15)$. The exponents average $0.39 \pm 0.05$ in ON cells and $0.50 \pm 0.05$ in OFF cells. These differences are consistent with previous results but are not statistically significant $(p>0.1)$.

\section{Model performance}

The model performs well in a vast majority of neurons, explaining overall $91.1 \%$ of the variance in the contrast-diameter experiments (median, $n=34$ ). This performance is remarkable, given that model parameters were estimated from a distinct data set (Fig. 3). To assess model performance in individual stimulus conditions, we computed $z$-scores of the deviations between measured and predicted responses. The amplitude of deviations is mostly below $1(|z|=0.65$, median; $n=34)$ indicating that, for most stimuli, model predictions approach the level of accuracy set by the trial-to-trial variability of responses.

The model predictions, however, showed small but significant biases. We estimated accuracy from the unsigned $z$-scores, conditioned on stimulus contrast or diameter. Predictions are most 

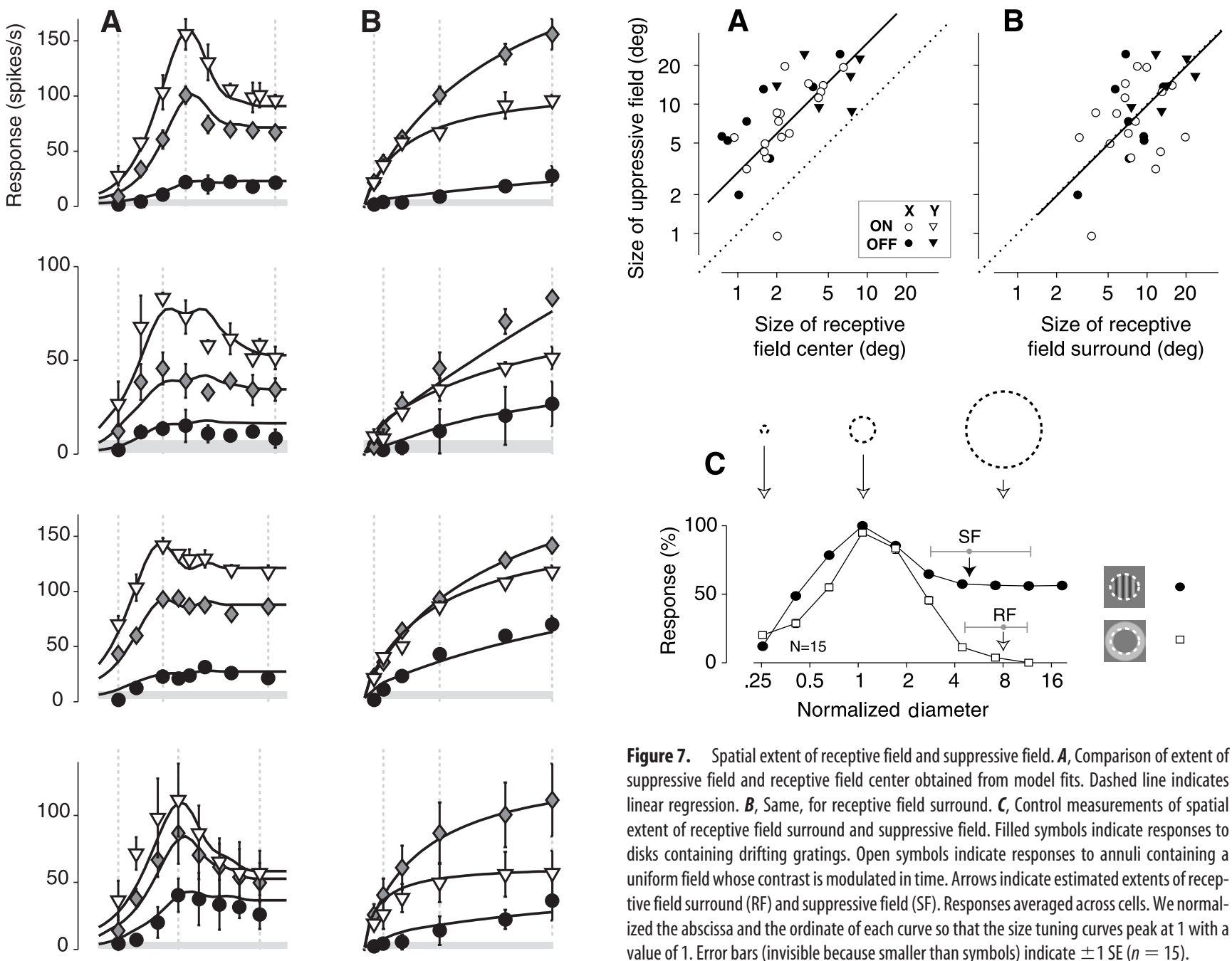

Figure 7. Spatial extent of receptive field and suppressive field. $\boldsymbol{A}$, Comparison of extent of suppressive field and receptive field center obtained from model fits. Dashed line indicates linear regression. $\boldsymbol{B}$, Same, for receptive field surround. $\boldsymbol{C}$, Control measurements of spatial extent of receptive field surround and suppressive field. Filled symbols indicate responses to disks containing drifting gratings. Open symbols indicate responses to annuli containing a uniform field whose contrast is modulated in time. Arrows indicate estimated extents of receptive field surround (RF) and suppressive field (SF). Responses averaged across cells. We normalized the abscissa and the ordinate of each curve so that the size tuning curves peak at 1 with a value of 1. Error bars (invisible because smaller than symbols) indicate $\pm 1 \mathrm{SE}(n=15)$.

$(|z|=0.86$ and 0.64$)$. We estimated bias from signed $z$-scores. The model overestimates the amplitudes of responses to large stimuli $(z=-0.71)$, whereas predictions for small stimuli show little bias $(z=-0.16)$. Likewise, the model overestimates the amplitudes of responses to low-contrast stimuli $(z=-0.89)$, whereas predictions for high-contrast stimuli show little bias $(z=$ $-0.06)$.

Because fit quality is good and biases are small, we conclude that our simple model of contrast gain control accounts for several suppressive phenomena observed in LGN responses. The model predicts contrast saturation, size tuning, and masking and explains how these phenomena are related to one another.

\section{Spatial extent}

Figure 6. Size tuning and contrast saturation in five additional cells. Details as in Figure 5. Gray areas indicate spontaneous response $\pm 1 \mathrm{SD}$. $\boldsymbol{A}$, Responses as a function of diameter, shown here for two contrasts: the lowest one eliciting a reliable response (black), $40 \%$ (gray), and $100 \%$ (white). $\boldsymbol{B}$, Responses expressed as a function of contrast, shown here for three diameters: the smallest diameter eliciting a reliable response (black), the optimal diameter (gray), and the largest diameter tested (white). Dotted lines indicate selected contrasts and diameters. Top to bottom, Cells 33.3.4 (0FF/X, 98.5\% explained variance), 28.2 .5 (ON/X, 91.8\%), 31.3.3 (OFF/X, 98.7\%), 35.3.3 (OFF/X, 93.7\%), and 31.2.2 (OFF/Y, 93.3\%).

accurate for diameters slightly larger than optimal $(|z|=0.52$, median) and least accurate for smaller and larger diameters $(|z|=$ 0.68 and 0.74$)$. Similarly, predictions are most accurate at $40 \%$ contrast $(|z|=0.52)$ and least accurate at 5 and $100 \%$ contrast
Studies in LGN suggested that the sources of suppression extend well beyond the classical receptive field (Levick et al., 1972; Murphy and Sillito, 1987; Cudeiro and Sillito, 1996; Felisberti and Derrington, 1999; Przybyszewski et al., 2000; Kaplan and Benardete, 2001; Sillito and Jones, 2002; Webb et al., 2002; Alitto and Usrey, 2003). How large is the suppressive field, and how does its spatial extent relate to that of the receptive field?

To our surprise, we found that suppressive field rarely extends beyond the surround of the classical receptive field (Fig. 7A,B). We obtained the extent of suppressive field and receptive field from model fits (Fig. 3). To compare the two, we concentrated on the 31 of 34 cells for which the receptive field surround was strong 
( $k_{\text {srd }}>0.2$; see Materials and Methods). The receptive fields of these cells had an average eccentricity of $13.9 \pm 9.1^{\circ}(\mathrm{SD})$. The suppressive field has an average extent of $10.3 \pm 6.6^{\circ}$. This is $\sim 300 \%$ larger than the receptive field center $\left(3.1 \pm 2.2^{\circ}\right.$ ) (Fig. $7 A)$ and approximately the same size as the receptive field surround $\left(9.1 \pm 5.1^{\circ}\right)($ Fig. $7 B)$.

The sizes of receptive field and suppressive field are strongly correlated across the population. The correlation between the sizes of receptive field center and suppressive field $(r=0.67 \pm$ 0.12 , bootstrap estimates) is comparable with the correlation between the sizes of receptive field center and surround $(r=0.53 \pm$ $0.16)$. The sizes of suppressive field and receptive field surround are also significantly correlated $(r=0.38 \pm 0.16)$.

These results seem discordant with previous studies, which describe suppression as originating beyond the classical receptive field (Levick et al., 1972; Murphy and Sillito, 1987; Cudeiro and Sillito, 1996; Felisberti and Derrington, 1999; Przybyszewski et al., 2000; Kaplan and Benardete, 2001; Sillito and Jones, 2002; Solomon et al., 2002; Webb et al., 2002; Alitto and Usrey, 2003). We therefore felt the need to confirm them with independent measurements (Fig. 7C). Indeed, the measures of receptive field extent presented above were obtained by fitting a difference-ofGaussians to responses to moving gratings of different spatial frequencies. Although this method is standard and has been thoroughly validated, it is indirect because it involves Fourier analysis.

To obtain a more direct measure of receptive field size, we recorded responses to contrast-modulated circular annuli $(n=$ 15 , in two additional animals) and varied annulus inner diameter (Fig. 7C, open symbols). The harmonic responses to these stimuli yield an annulus tuning curve that reflects the center-surround organization of the receptive field (Kilavik et al., 2003; Kremers et al., 2004). The inner diameter at which this curve reaches the floor (within \pm 1 SD of the response to a blank stimulus) indicates the size of the receptive field surround (Fig. $7 C$, open arrow). From the same cells, we also recorded responses to optimal drifting gratings and varied the diameter of the disk window (Fig. $7 C$, filled symbols). The diameter for which these curves reach a plateau (within \pm 1 SD of the response to the largest stimulus) indicates the size of the suppressive field (Fig. 7C, filled arrow). The results of these analyses confirm that, just as seen earlier using model parameters, the suppressive field does not extend beyond the region of the receptive field. On average, the estimated receptive field and suppressive field sizes were undistinguishable $(8.2 \pm 0.9$ and $7.9 \pm 1.9$ times larger than the optimal disk diameter, SE; $n=15)$. The normalized receptive field size had a median of 8.1 and a $50 \%$ confidence interval of 4.7-11.9 (Fig. 7C, open arrow and error bar). The normalized suppressive field size had a median of 4.6 and a confidence interval of $2.8-11.9$. Although the suppressive field may appear smaller than the receptive field, this difference is not statistically significant (Wilcoxon's rank sum test, $p>0.2$ ).

These findings confirm that the suppressive field is typically not larger than the surround of the classical receptive field and suggest an explanation for the discrepancy with the literature. Previous studies might have underestimated the actual extent of the receptive field and, in particular, of the receptive field surround. The surround only provides a weak drive to the cell, which can easily go unnoticed. Indeed, our results reveal that the method commonly used to estimate receptive field size systematically underestimates true size, typically by a remarkable factor of 8. Previous studies of LGN responses (Jones et al., 2000; Sillito and Jones, 2002; Solomon et al., 2002; Alitto and Usrey, 2003; Sun et al., 2004; Webb et al., 2005) have estimated the extent of the classical receptive field by measuring responses to high-contrast moving gratings windowed by disks of various sizes and finding the disk size that results in the maximal response. As we have seen (Fig. 7C), this measure underestimates the size of the receptive field surround by a factor of $8.2 \pm 0.9$. Given that the receptive field surround is approximately three times larger than the center (Table 1), this measure also underestimates the size of the receptive field center by a factor of 2.4. Given that previous studies were most likely underestimating the size of the receptive field surround, it is not surprising that they would describe the suppressive influences as originating outside the receptive field.

\section{Visual preferences}

We complete our characterization of gain control in LGN by studying the preferences of the suppressive field for spatial frequency, temporal frequency, and orientation. A quantitative assessment of these preferences provides constraints on the possible sources of suppressive signals (retina, thalamus, or cortex). It is also a necessary step toward a future application of the model to complex stimuli such as natural scenes.

As expected from previous qualitative assessments in retina and in LGN (Shapley and Victor, 1979; Cudeiro and Sillito, 1996; Sun et al., 2004), the suppressive field responds to a broader range of spatial frequencies than the receptive field (Fig. $8 A, B$ ). To estimate the tuning of receptive field and suppressive field for spatial frequency, we relied on model parameters obtained as described above. The tuning of the suppressive field is low pass in spatial frequency (Fig. $8 \mathrm{~B}$ ), with low-frequency cutoffs mostly below 0.01 cycles $/{ }^{\circ}$, the lowest spatial frequency tested. By comparison, the tuning of the receptive field has strong bandpass characteristics (Fig. 8A) and low-frequency cutoffs in the range of 0.05-0.2 cycles $^{\circ}{ }^{\circ}$ (Maffei and Fiorentini, 1973; Dawis et al., 1984; Cheng et al., 1995). The low-frequency cutoff of the suppressive field was only $0.23 \pm 0.12$ times that of the receptive field ( $n=34$, bootstrap estimates). The suppressive field also responds to frequencies that often extend beyond the resolution limit of the receptive field: on average, its high-frequency cutoff is $1.27 \pm$ 0.19 times that of the receptive field. Being broadly tuned, the suppressive field barely affects the spatial frequency selectivity of the neurons. Indeed, this selectivity depends only weakly on contrast. We measured spatial frequency tuning curves for stimuli at three contrast levels $(\sim 15,50$, and $100 \%)$ and calculated the high-frequency cutoff of each curve. The cutoff frequencies changed little with increasing contrast, from $0.33 \pm 0.04$ to $0.37 \pm 0.07$ to $0.39 \pm 0.07$ cycles $^{\circ}{ }^{\circ}$ (mean $\left.\pm \mathrm{SE} ; n=7\right)$. These values were not significantly different from one another (ANOVA, $p>0.7$ ). This contrast invariance of spatial frequency tuning seems consistent with the data, if not with the interpretation, of recent studies of spatial frequency tuning at different contrasts (Kremers et al., 2001; Nolt et al., 2004).

The suppressive field is broadly selective for temporal frequency and prefers frequencies of $10-20 \mathrm{~Hz}$ or higher (Fig. $8 C, D)$. To estimate the preferences of suppressive field and receptive field, we recorded responses to sums of test and mask gratings and varied mask temporal frequency. By measuring the reduction in test response caused by the different mask temporal frequencies, we estimated the temporal frequency tuning of the suppressive field. By measuring the response to the mask itself, we estimated the temporal frequency tuning of the receptive field. We obtained these measurements in 40 neurons (which include the 34 of our main sample). The suppressive field and receptive field exhibit similar selectivity: both respond weakly at low frequencies and strongly at high frequencies (Fig. $8 C, D$ ). These selectivity 
profiles resemble those obtained from responses to single drifting gratings varying in temporal frequency (Fig. $8 C$, dashed line) (Dawis et al., 1984; Saul and Humphrey, 1990; Alitto and Usrey, 2004). We did not explore frequencies above $21 \mathrm{~Hz}$ because, in our stimuli, test and mask were interleaved, and the refresh rate of the monitor was $124 \mathrm{~Hz}$. Therefore, we do not know how the suppressive field responds to very high frequencies.

This assessment of the selectivity of the suppressive field for temporal frequency complements previous studies of gain control in retina and LGN (Shapley and Victor, 1978, 1981; Benardete and Kaplan, 1999). These studies found that gain control affects responses to low frequencies and barely affects responses to high frequencies. Here, we concentrated on the responses to low frequencies, and we investigated the selectivity for temporal frequency of the suppressive field. Consistent with an early anecdotal report in retina (Shapley and Victor, 1979), we found this signal to be strong at high temporal frequencies but weak at low frequencies.

The suppressive field has no preferences for orientation: its bias for orientation is even weaker than that of the receptive field (Fig. 8E,F). To measure the orientation preferences of suppressive field and receptive field, we recorded responses to sums of test and mask gratings and varied mask orientation, following the same logic as the experiments in which we varied temporal frequency. We obtain these measurements in 30 of the 34 neurons in the main sample. The responses of the suppressive field are poorly tuned for orientation (Fig. $8 F$ ), reaching significance (ANOVA, $p<0.05$ ) in only 7 of 30 neurons. By comparison, the receptive field often shows consistent biases for orientation (Fig. 8E) (Vidyasagar and Urbas, 1982; Soodak et al., 1987; Shou and Leventhal, 1989; Sun et al., 2004), which reach significance (ANOVA, $p<0.05$ ) in 20 of 30 neurons. This bias had no consistent relationship with that seen occasionally in the suppressive field: aligning responses with the orientation that gave the peak receptive field response (Fig. $8 E$ ) and averaging across neurons yields a curve that is essentially flat (Fig. $8 F$ ). The suppressive field, therefore, is essentially insensitive to stimulus orientation.

This finding agrees with some reports (Solomon et al., 2002; Webb et al., 2002; Sun et al., 2004) but not with others (Sillito et al., 1993; Cudeiro and Sillito, 1996). The latter studies used gratings enclosed in a disk and an annulus and found that the orientation of the grating in the annulus affected the responses to the disk. We suggest that this result might be attributable to interactions between responses elicited by the disk and the annulus in the receptive field. The annulus in these studies was placed just outside the diameter that yields the maximal response for highcontrast disk stimuli. We have seen (Fig. 7C) that this diameter is approximately two times smaller than the receptive field center and eight times smaller than the receptive field surround. It is thus reasonable to assume that the annulus stimulated the receptive field surround and elicited some response of its own. This response would oscillate at the same frequency as the response to

\section{Receptive field}

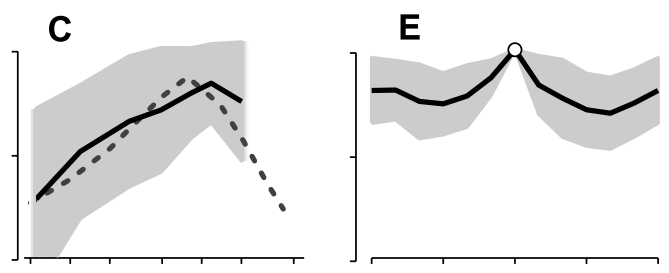

Suppressive field

D
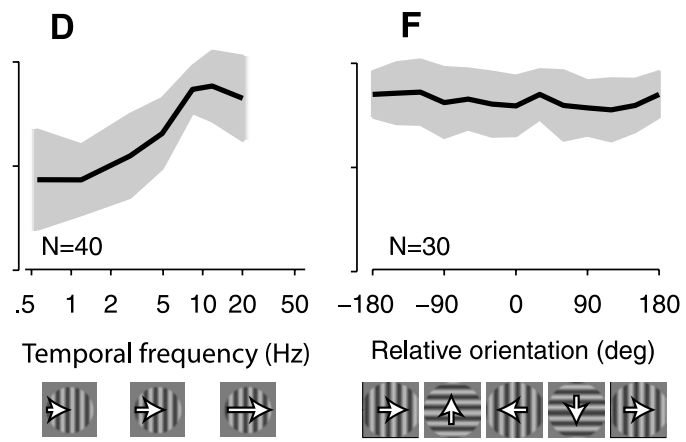

Figure 8. Visual preferences of receptive field and suppressive field. $\boldsymbol{A}$, Response of receptive field as function of spatial

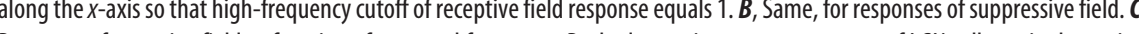
Responses were aligned so that maximum receptive field response is centered on $0^{\circ}$.

the disk. Depending on the details of the stimulus and of the receptive field, the two responses could interact destructively or constructively. The result could be an apparent effect of mask orientation.

In summary, we characterized the spatial extent and visual preferences of the suppressive field and found that it is generally (1) not larger than the receptive field surround, (2) broadly tuned for spatial frequency, (3) broadly selective for high temporal frequencies, and (4) not selective for orientation.

\section{Discussion}

We have formalized a simple model of gain control and have shown that it provides a parsimonious explanation for the diverse suppressive phenomena seen in LGN responses. The model predicts the phenomena of masking, size tuning, and contrast saturation and explains how these phenomena are interrelated. In particular, it explains how size tuning is pronounced at high contrast and weak at low contrast, and how increasing stimulus contrast reduces the size of the preferred stimulus. Furthermore, the model predicts a novel phenomenon: contrast saturation is present only for large stimuli, and responses to small stimuli grow almost linearly with contrast. This result indicates that there is no saturating nonlinearity at the output of LGN neurons: for stimuli that are small enough, LGN responses grow nearly linearly with contrast, with saturation being entirely explained in contrast gain control.

\section{Origins of the suppressive field}

Our measurements of the visual preferences of the suppressive field provide some constraints on the sources of suppression. The possible sources include the following: (1) mechanisms of suppression or gain control operating in retina (Cleland et al., 1983b; 
Kaplan et al., 1987; Sclar, 1987; Cheng et al., 1995; Girardin et al., 2002; Nolt et al., 2004); (2) inhibitory circuitry in thalamus (Hubel and Wiesel, 1961; Mcllwain and Creutzfeldt, 1967; Singer and Creutzfeldt, 1970; Levick et al., 1972; Cleland et al., 1983b; Cleland and Lee, 1985; Sherman and Koch, 1986; Kaplan et al., 1987; Funke and Eysel, 1998); and (3) negative feedback from visual cortex (Ahlsén and Lindström, 1983; Sherman and Koch, 1986; Murphy and Sillito, 1987; Sillito et al., 1993; Cudeiro and Sillito, 1996; Sillito and Jones, 2002; Webb et al., 2002; Worgotter et al., 2002; Alitto and Usrey, 2003).

The visual preferences of the suppressive field are entirely consistent with an origin in retina: just as the suppressive field, retinal ganglion cells respond as well to low spatial frequencies, follow high temporal frequencies, and are barely tuned for stimulus orientation. The mechanisms of contrast gain control in retina have been described by the work of Shapley and Victor (1978, 1981) and are beginning to be understood in terms of anatomy and biophysics (Chander and Chichilnisky, 2001; Kim and Rieke, 2001; Baccus and Meister, 2002; Demb, 2002; Zaghloul et al., 2005). To test whether the suppressive field in LGN is entirely inherited from retinal afferents, one could perform experiments with a test grating in one eye and a mask grating in the other eye.

Our measurements, however, cannot exclude a contribution from thalamic inhibition. There is evidence that gain control is stronger in LGN than in retina (Kaplan et al., 1987; Cheng et al., 1995). LGN cells receive inhibition from two sources (Dubin and Cleland, 1977; Ahlsén et al., 1982; Sherman and Koch, 1986): feedforward from local interneurons and feedback from the perigeniculate nucleus (PGN). Interneurons have visual properties that resemble the properties of relay cells (Dubin and Cleland, 1977). It might therefore not be trivial to obtain a broad tuning for spatial frequency by pooling the outputs of interneurons. A more likely source of suppression lies in PGN neurons, whose visual preferences resemble those of the suppressive field. PGN neurons (1) have large receptive fields (Sanderson, 1971; Uhlrich et al., 1991; Wrobel and Bekisz, 1994; Funke and Eysel, 1998), (2) respond well to low spatial frequencies (Price and Morgan, 1987; Xue et al., 1988; Murphy et al., 1994; Wrobel and Bekisz, 1994), (3) respond equally to light increments and decrements (ON/ OFF responses) (Sanderson, 1971; Dubin and Cleland, 1977; So and Shapley, 1981; Xue et al., 1988; Uhlrich et al., 1991; Wrobel and Bekisz, 1994; Funke and Eysel, 1998), and (4) show poor selectivity for stimulus size (Jones and Sillito, 1994). The last property is consistent with the observation that suppression is maintained for large diameters (Fig. 3B).

Finally, the visual preferences of the suppressive field are hardly consistent with a cortical origin. The suppressive field has the same extent as the receptive field surround, whereas neurons in primary visual cortex (V1) have larger receptive fields (Jones et al., 2000). More importantly, the suppressive field responds strongly to low spatial frequencies (Fig. $8 B$ ) and high temporal frequencies (Fig. 8D), which elicit little response in V1 neurons (Ikeda and Wright, 1975; Movshon et al., 1978; Saul and Humphrey, 1992; DeAngelis et al., 1993; Freeman et al., 2002).

Cortical feedback, however, does seem to affect LGN responses (Alitto et al., 2002; Sillito and Jones, 2002; Worgotter et al., 2002). Cortical ablation or inactivation modifies the gain of the neurons (Przybyszewski et al., 2000; Webb et al., 2002) and reduces size tuning (Murphy and Sillito, 1987; Alitto and Usrey, 2003). These effects might possibly be explained if cortex modulated the strength of the suppressive field, for example by controlling the value of the parameter $c_{50}$, which we have considered to be constant (Fig. $3 A, B$ ).

\section{Limitations of our study}

A limitation of our study is that we did not consider the nonlinear spatial summation seen in both retina and LGN. We have modeled the component of the responses oscillating at the stimulus frequency. This component accounts for most of the variance in the responses of X cells. Y cells, however, also respond to moving gratings with an elevated mean firing rate (Enroth-Cugell and Robson, 1966; Hochstein and Shapley, 1976; Derrington et al., 1979; Demb et al., 1999). We did not consider the mean rate of responses and therefore could not test the suggestion that the subunits underlying the responses of Y cells correspond to the bank of filters in the suppressive field (Shapley and Victor, 1978, 1981; Victor, 1987).

Another example of nonlinear summation that we have not considered is the "shift effect" (also known as the "periphery effect"), whereby the firing rate of a neuron can be increased by stimulus moving well outside the receptive field (McIlwain and Creutzfeldt, 1964; Levick et al., 1965; Ikeda and Wright, 1972; Kruger and Fischer, 1973; Derrington et al., 1979; Passaglia et al., 2001). Our model cannot explain this increase. Remote stimulation, however, can also induce suppressive effects that have been related to the shift effect (Felisberti and Derrington, 1999, 2001; Passaglia et al., 2001; Girardin et al., 2002). These effects resemble the effects of the suppressive field in many aspects. First, remote stimulation has a divisive effect on the responses to a central target (Felisberti and Derrington, 1999). Second, it affects the contrast gain of the neurons (Felisberti and Derrington, 1999; Girardin et al., 2002). Third, suppression can be induced by stimuli as distant as $\sim 10^{\circ}$ away from the receptive field center. This finding might be consistent with our finding that the suppressive field extends over a large region of visual field $\left(10.3 \pm 6.6^{\circ}\right.$ in diameter at an average eccentricity of $13.9 \pm 9.1^{\circ}$ ).

A major limitation of our work is that we have not considered the time course of the responses. Contrast gain control not only affects the gain of the responses but also their temporal dynamics (Shapley and Victor, 1978, 1981). Increasing contrast reduces the integration time of the neurons affecting the phase of responses and their selectivity for temporal frequency. The model does not capture these effects: in fact, we intentionally simplified the Shapley-Victor model so that it would predict only the amplitude of the responses. Our model therefore only works for stimuli of fixed temporal frequencies.

Moreover, we have not considered the dynamics of contrast gain control (i.e., how a change in contrast at a given time can influence gain at a later time). We cannot study these dynamics because, in our stimuli, contrast was fixed through time. In particular, we cannot distinguish the fast mechanism of contrast gain control (Victor, 1987; Baccus and Meister, 2002; Mante et al., 2005; Zaghloul et al., 2005) from the slower mechanism of contrast adaptation (Chander and Chichilnisky, 2001; Kim and Rieke, 2001; Baccus and Meister, 2002; Solomon et al., 2004). We suspect that slow adaptation plays little role in our results, because it is pronounced only for high-frequency stimuli (Solomon et al., 2004) and is weak at lower frequencies (Ohzawa et al., 1985; Shou et al., 1996; Sanchez-Vives et al., 2000; Yang et al., 2003; Solomon et al., 2004). Moreover, our stimuli were brief (typically $1-2 \mathrm{~s}$, always $<4 \mathrm{~s}$ ), presented in randomized order, and separated by $\sim 1$ s of blank screen; these properties would minimize the effects of slow adaptation, which take seconds to develop and are strongest when low contrast stimuli are preceded by long periods of strong stimulation (Solomon et al., 2004). 


\section{Implications for $\mathrm{V} 1$ responses}

Our formulation of retinal contrast gain control in terms of division reveals close similarities with models of gain control or normalization in primary visual cortex (Albrecht and Geisler, 1991; Heeger, 1992; Carandini et al., 1997). These models predict expressions similar to Equation 6. In fact, neurons in primary visual cortex exhibit contrast saturation (Albrecht and Hamilton, 1982; Sclar et al., 1990), masking (Bonds, 1989; Freeman et al., 2002), and size tuning (Sceniak et al., 1999; Cavanaugh et al., 2002). The exact contribution of LGN suppression to these phenomena is not clear. It is evident that some cortical effects, such as the orientation selectivity of surround suppression (Cavanaugh et al., 2002), are not easily explained by suppression in LGN. Nonetheless, our results indicate that the suppressive phenomena seen in LGN would be expected to contribute to those seen in V1.

The fact that a model with similar structure can explain gain control in retina, in LGN, and in cortex is very good news. It paves the way for a long-due assessment of the relative roles of these stages of visual processing to phenomena of suppression, gain control, and normalization. Once these phenomena are fitted with the same model in the different stages, then the contribution of each stage can be described by the parameters of the model (Sclar, 1987; Sclar et al., 1990).

\section{Function of the suppressive field}

The suppressive field is distinct from the receptive field in many respects. First, it is responsible for suppressive phenomena that cannot be explained by the receptive field alone (Fig. 3, dashed lines). Second, it responds well to high and low spatial frequencies (Fig. $8 B$ ), frequencies that generally elicit poor responses in the receptive field (Fig. $8 \mathrm{~A}$ ). Third, it computes the SD of stimulus intensities (Fig. 1).

What use might the visual system make of divisive suppression? One possible use is to map the wide range of contrasts encountered in the natural environment onto the limited dynamic range of the neurons (Heeger, 1992). Suppression may also play a role in encoding of visual information by populations of neurons: a divisive gain control model applied to responses in primary visual cortex was shown to increase independence of the responses across neurons (Schwartz and Simoncelli, 2001; Valerio and Navarro, 2003).

We suggest that the suppressive field could also play a role in the joint encoding of spatial frequency and spatial position, which are subject to a stringent tradeoff (Daugman, 1985). In LGN, selectivity for spatial frequency results from the antagonistic effect of the receptive field center and surround. This selectivity comes, however, at the cost of lower spatial localization because of the large size of the receptive field surround. The suppressive field counteracts this effect by reducing the effective integration area of the neuron. This improvement in encoding of spatial position comes at no cost because the suppressive field barely affects the tuning of responses for spatial frequency (Fig. $3 F)$.

\section{Toward a functional model of LGN neurons}

Our model is a parsimonious description of the processing performed by LGN neurons on spatial images. It features few free parameters, each of which has a clear functional interpretation. These parameters can be reliably estimated from a limited set of measurements and held fixed to predict data obtained in new experiments.

This research, thus, brings us closer to a central goal of visual neuroscience, the development of functional models of neural responses to visual stimuli. Functional models summarize the image processing performed by neural circuits, guide research in the underlying biophysics, and facilitate the study of subsequent visual stages. Functional models are particularly desirable for LGN, whose responses reflect the complex computations performed in retina, and constitute the principal input to the visual cortex. As the first inroads toward thalamic visual prostheses are being made (Pezaris and Reid, 2004), the need for such a model may become much more pressing.

\section{Appendix: divisive effect of gain control}

Here we show that the model of retinal contrast gain control by Shapley and Victor (1981) predicts that contrast has a divisive effect on the responses. The model consists of a fixed low-pass filter followed by a high-pass stage whose time constant varies with contrast.

For simplicity, we consider the version of their model described by Victor (1987), in which responses to a stimulus with contrast $c$ and temporal frequency $\omega /(2 \pi)$ are given by the following:

$$
R(c, \omega)=c L(\omega)\left(1-\frac{h}{1+i \omega \tau(c)}\right),
$$

where $L(\omega)$ is a low-pass filter, and the expression in the large parentheses is a high-pass filter with gain $h$ and time constant $\tau(c)$. Following Victor (1987), we take the latter to depend on contrast $c$ as follows:

$$
\tau(c)=\frac{\tau_{0}}{1+\left(c / c_{50}\right)^{n}}
$$

with $n, \tau_{0}, c_{50}$ constants.

Fixing $h=1$ (Benardete and Kaplan, 1999), we substitute Equation 8 into Equation 7 and calculate the absolute value to obtain the following:

$$
|R(c, \omega)|=|L(\omega)| \frac{c}{\sqrt{\left(c_{50}^{n}+c^{n}\right)^{2}+\omega^{2} \tau_{0}^{2} c_{50}^{2 n}}},
$$

where $|L(\omega)|$ is the amplitude of the linear response, and the right-hand term captures the effect of gain control. The denominator in Equation 9 is responsible for the effects of gain control. Gain control is pronounced at low temporal frequencies $(\omega \ll$ $1 / \tau_{0}$ ) for which the denominator grows with contrast. Gain control is absent at high frequencies $\left(\omega \gg 1 / \tau_{0}\right)$ for which the denominator becomes independent of contrast. With $\omega$ close to 0 , Equation 9 reduces to a hyperbolic ratio:

$$
|R(c)|=|L(0)| \frac{c}{\left(c_{50}^{n}+c^{n}\right)},
$$

which demonstrates that contrast gain control in retina is divisive, because contrast appears not only in the numerator but also in the denominator.

In cat X ganglion cells, $\tau_{0}$ has a median value of $0.23 \mathrm{~s}$ (Victor, 1987), so the approximation provided by Equation 9 is exact only for frequencies less than $1 / 0.23 /(2 \pi)=0.69 \mathrm{~Hz}$. However, we found that the expression provides good fits to responses at frequencies as high as $12 \mathrm{~Hz}$.

Our Equation 6 follows from Equation 10 once one sets $n=1$. Indeed, this value seems more consistent with the data (Fig. 3) (Sclar et al., 1990) than the value $n=2$ used by Victor (1987). 


\section{References}

Ahlsén G, Lindström S (1983) Corticofugal projection to perigeniculate neurones in the cat. Acta Physiol Scand 118:181-184.

Ahlsén G, Lindström S, Lo FS (1982) Functional distinction of perigeniculate and thalamic reticular neurons in the cat. Exp Brain Res 46:118-126.

Albrecht DG, Geisler WS (1991) Motion sensitivity and the contrastresponse function of simple cells in the visual cortex. Vis Neurosci 7:531-546.

Albrecht DG, Hamilton DB (1982) Striate cortex of monkey and cat: contrast response function. J Neurophysiol 48:217-237.

Alitto HJ, Usrey WM (2003) Corticothalamic feedback and sensory processing. Curr Opin Neurobiol 13:440-445.

Alitto HJ, Usrey WM (2004) The influence of contrast on orientation and temporal frequency tuning in ferret primary visual cortex. J Neurophysiol 91:2797-2808.

Alitto HJ, Collins OA, Usrey WM (2002) The influence of corticothalamic feedback on the responses of neurons in lateral geniculate nucleus. Soc Neurosci Abstr 28:658.16.

Baccus SA, Meister M (2002) Fast and slow contrast adaptation in retinal circuitry. Neuron 36:909-919.

Benardete EA, Kaplan E (1999) The dynamics of primate M retinal ganglion cells. Vis Neurosci 16:355-368.

Bonds AB (1989) Role of inhibition in the specification of orientation selectivity of cells in the cat striate cortex. Vis Neurosci 2:41-55.

Brainard DH (1997) The psychophysics toolbox. Spat Vis 10:433-436.

Carandini M, Heeger DJ, Movshon JA (1997) Linearity and normalization in simple cells of the macaque primary visual cortex. J Neurosci 17:8621-8644.

Cavanaugh JR, Bair W, Movshon JA (2002) Nature and interaction of signals from the receptive field center and surround in macaque v1 neurons. J Neurophysiol 88:2530-2546.

Chander D, Chichilnisky EJ (2001) Adaptation to temporal contrast in primate and salamander retina. J Neurosci 21:9904-9916.

Cheng H, Chino YM, Smith III EL, Hamamoto J, Yoshida K (1995) Transfer characteristics of lateral geniculate nucleus X neurons in the cat: effects of spatial frequency and contrast. J Neurophysiol 74:2548-2557.

Chino YM, Kaplan E (1988) Abnormal orientation bias of LGN neurons in strabismic cats. Invest Ophthalmol Vis Sci 29:644-648.

Cleland BG, Lee BB (1985) A comparison of visual responses of cat lateral geniculate nucleus neurones with those of ganglion cells afferent to them. J Physiol (Lond) 369:249-268.

Cleland BG, Lee BB, Vidyasagar TR (1983a) Response of neurons in the cat's lateral geniculate nucleus to moving bars of different length. J Neurosci 3:108-116.

Cleland BG, Harding TH, Tulunay-Keesey U (1983b) Response to the length of moving visual stimuli of the brisk classes of ganglion cells in the cat retina. J Physiol (Lond) 345:27-45.

Cudeiro J, Sillito AM (1996) Spatial frequency tuning of orientationdiscontinuity-sensitive corticofugal feedback to the cat lateral geniculate nucleus. J Physiol (Lond) 490:481-492.

Daugman JG (1985) Uncertainty relation for resolution in space, spatial frequency, and orientation optimized by two-dimensional visual cortical filters. J Opt Soc Am A 2:1160-1169.

Dawis S, Shapley R, Kaplan E, Tranchina D (1984) The receptive field organization of $\mathrm{x}$ cells in the cat spatiotemporal coupling and asymmetry. Vision Res 24:549-564.

DeAngelis GC, Ohzawa I, Freeman RD (1993) Spatiotemporal organization of simple-cell receptive fields in the cat's striate cortex. II. Linearity of temporal and spatial summation. J Neurophysiol 69:1118-1135.

Demb JB (2002) Multiple mechanisms for contrast adaptation in the retina. Neuron 36:781-783.

Demb JB, Haarsma L, Freed MA, Sterling P (1999) Functional circuitry of the retinal ganglion cell's nonlinear receptive field. J Neurosci 19:9756-9767.

Derrington AM, Lennie P (1984) Spatial and temporal contrast sensitivities of neurons in lateral geniculate nucleus of macaque. J Physiol (Lond) 357:219-240.

Derrington AM, Lennie P, Wright MJ (1979) The mechanism of peripherally evoked responses in retinal ganglion cells. J Physiol (Lond) 289:299-310.

Dubin MW, Cleland BG (1977) Organization of visual inputs to interneurons of lateral geniculate nucleus of the cat. J Neurophysiol 40:410-427.
Enroth-Cugell C, Robson JG (1966) The contrast sensitivity of retinal ganglion cells of the cat. J Physiol (Lond) 187:517-552.

Felisberti F, Derrington AM (1999) Long-range interactions modulate the contrast gain in the lateral geniculate nucleus of cats. Vis Neurosci 16:943-956.

Felisberti F, Derrington AM (2001) Long-range interactions in the lateral geniculate nucleus of the New-World monkey, Callithrix jacchus. Vis Neurosci 18:209-218.

Freeman T, Durand S, Kiper D, Carandini M (2002) Suppression without inhibition in visual cortex. Neuron 35:759-771.

Funke K, Eysel UT (1998) Inverse correlation of firing patterns of single topographically matched perigeniculate neurons and cat dorsal lateral geniculate relay cells. Vis Neurosci 15:711-729.

Girardin CC, Kiper DC, Martin KA (2002) The effect of moving textures on the responses of cells in the cat's dorsal lateral geniculate nucleus. Eur J Neurosci 16:2149-2156.

Heeger DJ (1992) Normalization of cell responses in cat striate cortex. Vis Neurosci 9:181-197.

Hochstein S, Shapley RM (1976) Linear and nonlinear spatial subunits in Y cat retinal ganglion cells. J Physiol (Lond) 262:265-284.

Hubel D, Wiesel TN (1961) Integrative action in the cat's lateral geniculate body. J Physiol (Lond) 155:385-398.

Ikeda H, Wright MJ (1972) The periphery effect and its relation to the receptive field organization of "transient" retinal ganglion cells. J Physiol (Lond) 226:81P-82P.

Ikeda H, Wright MJ (1975) Spatial and temporal properties of "sustained" and "transient" neurones in area 17 of the cat's visual cortex. Exp Brain Res 22:363-383.

Jones HE, Sillito AM (1991) The length-response properties of cells in the feline dorsal lateral geniculate nucleus. J Physiol (Lond) 444:329-348.

Jones HE, Sillito AM (1994) The length-response properties of cells in the feline perigeniculate nucleus. Eur J Neurosci 6:1199-1204.

Jones HE, Andolina IM, Oakely NM, Murphy PC, Sillito AM (2000) Spatial summation in lateral geniculate nucleus and visual cortex. Exp Brain Res 135:279-284.

Kaplan E, Benardete E (2001) The dynamics of primate retinal ganglion cells. Prog Brain Res 134:17-34.

Kaplan E, Purpura K, Shapley R (1987) Contrast affects the transmission of visual information through the mammalian lateral geniculate nucleus. J Physiol (Lond) 391:267-288.

Kilavik BE, Silveira LC, Kremers J (2003) Centre and surround responses of marmoset lateral geniculate neurones at different temporal frequencies. J Physiol (Lond) 546:903-919.

Kim KJ, Rieke F (2001) Temporal contrast adaptation in the input and output signals of salamander retinal ganglion cells. J Neurosci 21:287-299.

Kremers J, Silveira LC, Kilavik BE (2001) Influence of contrast on the responses of marmoset lateral geniculate cells to drifting gratings. J Neurophysiol 85:235-246.

Kremers J, Kozyrev V, Silveira LC, Kilavik BE (2004) Lateral interactions in the perception of flicker and in the physiology of the lateral geniculate nucleus. J Vis 4:643-663.

Kruger J, Fischer B (1973) Strong periphery effect in cat retinal ganglion cells. Excitatory responses in ON- and OFF-center neurones to single grid displacements. Exp Brain Res 18:316-318.

Levick WR, Oyster CW, Davis DL (1965) Evidence that Mcilwain's periphery effect is not a stray light artifact. J Neurophysiol 28:555-559.

Levick WR, Cleland BG, Dubin MW (1972) Lateral geniculate neurons of cat: retinal inputs and physiology. Invest Ophthalmol 11:302-311.

Maffei L, Fiorentini A (1973) The visual cortex as a spatial frequency analyzer. Vis Res 13:1255-1267.

Mante V, Frazor RA, Bonin V, Geisler WS, Carandini M (2005) Independence of luminance and contrast in natural images and in the early visual system. Nat Neurosci, in press.

McIlwain JT, Creutzfeldt OD (1964) Receptive fields of optic tract axons and lateral geniculate cells: peripheral extent and barbiturate sensitivity. J Neurophysiol 27:1154-1173.

McIlwain JT, Creutzfeldt OD (1967) Microelectrodes study of synaptic excitation and inhibition in the lateral geniculate nucleus of the cat. J Neurophysiol 30:1-21.

Movshon JA, Thompson ID, Tolhurst DJ (1978) Spatial and temporal contrast sensitivity of neurones in areas 17 and 18 of the cat's visual cortex. J Physiol (Lond) 283:101-120. 
Murphy PC, Sillito AM (1987) Corticofugal feedback influences the generation of length tuning in the visual pathway. Nature 329:727-729.

Murphy PC, Uhlrich DJ, Tamamaki N, Sherman SM (1994) Brain-stem modulation of the response properties of cells in the cat's perigeniculate nucleus. Vis Neurosci 11:781-791.

Nolt MJ, Kumbhani RD, Palmer LA (2004) Contrast-dependent spatial summation in the lateral geniculate nucleus and retina of the cat. J Neurophysiol 92:1708-1717.

Ohzawa I, Sclar G, Freeman RD (1985) Contrast gain control in the cat visual system. J Neurophysiol 54:651-665.

Ozeki H, Sadakane O, Akasaki T, Naito T, Shimegi S, Sato H (2004) Relationship between excitation and inhibition underlying size tuning and contextual response modulation in the cat primary visual cortex. J Neurosci 24:1428-1438.

Passaglia CL, Enroth-Cugell C, Troy JB (2001) Effects of remote stimulation on the mean firing rate of cat retinal ganglion cells. J Neurosci 21:5794-5803.

Pelli DG (1997) The VideoToolbox software for visual psychophysics: transforming numbers into movies. Spat Vis 10:437-442.

Pezaris JS, Reid RC (2004) Microstimulation in LGN produces focal visual percepts. Soc Neurosci Abstr 30:598.5.

Price DJ, Morgan JE (1987) Spatial properties of neurones in the lateral geniculate nucleus of the pigmented ferret. Exp Brain Res 68:28-36.

Przybyszewski AW, Gaska JP, Foote W, Pollen DA (2000) Striate cortex increases contrast gain of macaque LGN neurons. Vis Neurosci $17: 485-494$.

Rodieck RW (1965) Quantitative analysis of cat retina ganglion cell response to visual stimuli. Vision Res 5:583-601.

Sanchez-Vives MV, Nowak LG, McCormick DA (2000) Membrane mechanisms underlying contrast adaptation in cat area 17 in vivo. J Neurosci 20:4267-4285.

Sanderson KJ (1971) The projection of the visual field to the lateral geniculate and medial interlaminar nuclei in the cat. J Comp Neurol 143:101-108.

Saul AB, Humphrey AL (1990) Spatial and temporal response properties of lagged and nonlagged cells in cat lateral geniculate nucleus. J Neurophysiol 64:206-224.

Saul AB, Humphrey AL (1992) Temporal-frequency tuning of direction selectivity in cat visual cortex. Vis Neurosci 8:365-372.

Sceniak MP, Ringach DL, Hawken MJ, Shapley R (1999) Contrast's effect on spatial summation by macaque V1 neurons. Nat Neurosci 2:733-739.

Schwartz O, Simoncelli EP (2001) Natural signal statistics and sensory gain control. Nat Neurosci 4:819-825.

Sclar G (1987) Expression of "retinal" contrast gain control by neurons of the cat's lateral geniculate nucleus. Exp Brain Res 66:589-596.

Sclar G, Maunsell JHR, Lennie P (1990) Coding of image contrast in central visual pathways of the macaque monkey. Vision Res 30:1-10.

Shapley R, Victor JD (1979) Nonlinear spatial summation and the contrast gain control of cat retinal ganglion cells. J Physiol (Lond) 290:141-161.

Shapley RM, Victor JD (1978) The effect of contrast on the transfer properties of cat retinal ganglion cells. J Physiol (Lond) 285:275-298.

Shapley RM, Victor J (1981) How the contrast gain modifies the frequency responses of cat retinal ganglion cells. J Physiol (Lond) 318:161-179.

Sherman SM, Koch C (1986) The control of retinogeniculate transmission in the mammalian lateral geniculate nucleus. Exp Brain Res 63:1-20.

Shou T, Li X, Zhou Y, Hu B (1996) Adaptation of visually evoked responses of relay cells in the dorsal lateral geniculate nucleus of the cat following prolonged exposure to drifting gratings. Vis Neurosci 13:605-613.
Shou TD, Leventhal AG (1989) Organized arrangement of orientationsensitive relay cells in the cat's dorsal lateral geniculate nucleus. J Neurosci 9:4287-4302.

Sillito AM, Jones HE (2002) Corticothalamic interactions in the transfer of visual information. Philos Trans R Soc Lond B Biol Sci 357:1739-1752.

Sillito AM, Cudeiro J, Murphy PC (1993) Orientation sensitive elements in the corticofugal influence on centre-surround interactions in the dorsal lateral geniculate nucleus. Exp Brain Res 93:6-16.

Singer W, Creutzfeldt OD (1970) Reciprocal lateral inhibition of on- and off-center neurones in the lateral geniculate body of the cat. Exp Brain Res 10:311-330.

So YT, Shapley R (1981) Spatial tuning of cells in and around lateral geniculate nucleus of the cat $\mathrm{x}$ and $\mathrm{y}$ relay cells and peri geniculate inter neurons. J Neurophysiol 45:107-120.

Solomon SG, White AJ, Martin PR (2002) Extraclassical receptive field properties of parvocellular, magnocellular, and koniocellular cells in the primate lateral geniculate nucleus. J Neurosci 22:338-349.

Solomon SG, Peirce JW, Dhruv NT, Lennie P (2004) Profound contrast adaptation early in the visual pathway. Neuron 42:155-162.

Soodak RE, Shapley RM, Kaplan E (1987) Linear mechanism of orientation tuning in the retina and lateral geniculate nucleus of the cat. J Neurophysiol 58:267-275.

Sun C, Chen X, Huang L, Shou T (2004) Orientation bias of the extraclassical receptive field of the relay cells in the cat's dorsal lateral geniculate nucleus. Neuroscience 125:495-505.

Uhlrich DJ, Cucchiaro JB, Humphrey AL, Sherman SM (1991) Morphology and axonal projection patterns of individual neurons in the cat perigeniculate nucleus. J Neurophysiol 65:1528-1541.

Valerio R, Navarro R (2003) Input-output statistical independence in divisive normalization models of V1 neurons. Network 14:733-745.

Victor J (1987) The dynamics of the cat retinal X cell centre. J Physiol (Lond) 386:219-246.

Vidyasagar TR, Urbas JV (1982) Orientation sensitivity of cat LGN neurones with and without inputs from visual cortical areas 17 and 18. Exp Brain Res 46:157-169.

Webb BS, Tinsley CJ, Barraclough NE, Easton A, Parker A, Derrington AM (2002) Feedback from V1 and inhibition from beyond the classical receptive field modulates the responses of neurons in the primate lateral geniculate nucleus. Vis Neurosci 19:583-592.

Webb BS, Tinsley CJ, Vincent CJ, Derrington AM (2005) Spatial distribution of suppressive signals outside the classical receptive-field in lateral geniculate nucleus. J Neurophysiol 94:1789-1797.

Worgotter F, Eyding D, Macklis JD, Funke K (2002) The influence of the corticothalamic projection on responses in thalamus and cortex. Philos Trans R Soc Lond B Biol Sci 357:1823-1834.

Wrobel A, Bekisz M (1994) Visual classification of X and Y perigeniculate neurons of the cat. Exp Brain Res 101:307-313.

Xue JT, Carney T, Ramoa AS, Freeman RD (1988) Binocular interaction in the perigeniculate nucleus of the cat. Exp Brain Res 69:497-508.

Yang Y, Jin J, Zhou Y, Shou T (2003) GABA(A) and GABA(B) receptors mediated inhibition affect the pattern adaptation of relay cells in the dorsal lateral geniculate nucleus (LGNd) of cats. Brain Res 959:295-303.

Zaghloul KA, Boahen K, Demb JB (2003) Different circuits for ON and OFF retinal ganglion cells cause different contrast sensitivities. J Neurosci 23:2645-2654.

Zaghloul KA, Boahen K, Demb JB (2005) Contrast adaptation in subthreshold and spiking responses of mammalian Y-type retinal ganglion cells. J Neurosci 25:860-868. 